\title{
ON THE PENETRATION OF NUCLEONS THROUGH HEAVY NUCLEI
}

\author{
by G. C. J. ZWANIKKEN \\ Instituut voor Theoretische Natuurkunde der Rijks-Universitcit, Litrecht
}

\section{Summary}

The rate of loss of energy of a nucleon traversing a nucleus and the mechanism of star production are investigated on the basis of the meson type of interaction $e^{-\kappa r} / \kappa r$. The theoretical predictions are compared with the available empirical data.

I. Introduction. The microscopic examination of specially prepared photographic emulsions, which have been exposed to cosmic radiation, is an important means of studying nuclear disintegration processes. These processes manifest themselves in the emulsion as disintegration-stars, which arise from the disintegration of a nucleus into many particles by a collision with a cosmic particle, which we shall assume to be a neutron. Of course, only charged particles are recorded. From the length of path in the emulsion, the variation of grain density and the Coulomb scattering, it is possible to draw important conclusions about the nature and energy of the knockedout particles. These energies may exceed $100 \mathrm{MeV}$.

It appears that protons are most frequent. So one has to assume that on the average an equal number of neutrons must be expelled. One arrives therefore at the conclusion that the energy, transmitted from the impinging particle to the nucleus, is at least twice the sum of the proton energies, i.e. of the order of a few hundreds of $\mathrm{MeV}$. Larger energies are, however, also frequent.

For the Gauss potential, $\mathrm{H}$ e i s e $\mathrm{n}$ be r $\mathrm{g}$ (1937) and B a g g e (1939) have given computations of the energy distribution of the knocked-out nucleons and the maximum energy loss of the impinging neutron. From the energy distribution one may draw conclu- 
sions about the magnitude of the range $x^{-1}$ of the nuclear potential. The strength of the potential does not appear in the equation for the relative energy distribution. Ortner (1940) compared the computed values with the experimental data and found a good agreement. It is therefore desirable to perform the calculations also for the meson potential.

There are, however, some differences with the corresponding calculations for the Gauss potential. First the less rapid falling off of the meson potential leads to a considerable number of high energy particles. Although the experimental results of Ortner (1940) are in good agreement with the calculations using the Gauss potential, the more recent data of Pe r kin s (1947) indicate that the number of high energy particles is too high to be explained by a Gauss potential. Taking into account, however, the unrecorded protons, the agreement of the figures for the meson potential with the most reliable experimental data is very satisfactory indeed, if $z^{-1}=2 \times 10^{-13} \mathrm{~cm}$.

When computing the energy loss per unit path in nuclear matter, it is necessary to use the relativistic expression for the kinetic energy, because otherwise this expression will contain a divergent integral. Then the upper limit for the mean energy loss per collision is $31 \mathrm{MeV}$.

Before the computation some remarks are given about the collision cross section. These remarks refer to the way in which the Pauli principle is taken in account. It appears that the formulae must be altered slightly. For the results of $\mathrm{H}$ e is e $\mathrm{nber} g$ and $\mathrm{B}$ a g g e these alterations are only of little importance.

II. The collision cross section. In this section we shall discuss the collision cross section only so far as is necessary to bring about the changes to be made in the theory of $\mathrm{H}$ e is e $\mathrm{nberg}$. For the derivation of the formulae we refer to the book of $\mathrm{R}$ os e $\mathrm{n} f$ eld whose notations we shall also use. All calculations are made with the help of the meson potential $e^{-\kappa r} / \varkappa r$, without using meson field theory, and neglecting the electrostatic interaction of the nucleons. We shall assume that we may represent the heavy nucleus by a Fermi gas of the composing nucleons. For the collision cross section we take the formula derived with the Born approximation. Then the 
equation for the differential collision cross section of a neutron with one of the particles of the nucleus, reads

$$
d S=\left.\left(c / v_{i}^{(1)}\right)(2 \pi V / \hbar) !\left(i \mid U_{\text {nacl }} ! f\right)\right|^{2} d n_{f},
$$

with $v_{i}^{(1)}$ the velocity of the impinging neutron. The indices (1) and (2) refer to the impinging and knocked-on particles respectively and the indices $(i)$ and $(f)$ refer to the initial and final states; $V$ is the volume of the nucleus, $\left(i\left|U_{\text {nucl }}\right| f\right)$ the matrix element of the nuclear potential and $d n$, the number of stationary states of the system per unit energy after collision. It is necessary to take into account the identity of impinging and knocked particles with respect to the Pauli principle. After averaging over spin and charge variables, we have:

$$
\left|\left(i\left|U_{\text {nucl }}\right| f\right)\right|^{2}=0.52 \frac{J^{2}}{V^{2}} \frac{4 \pi^{2}}{c^{2} x^{6}}\left[\left(\frac{2}{1+(p / \hbar x)^{2}}\right)^{2}+\left(\frac{2}{1+\left(p^{\prime} / \hbar x\right)^{2}}\right)^{2}\right] ;
$$

$J$ is the strength of the meson potential with range $x^{-1}$ and $p, p^{\prime}$ are the magnitudes of the momentum transfers in the collision:

$$
\begin{aligned}
\mathbf{p} & =\mathbf{p}_{i}^{(1)}-\mathbf{p}_{f}^{(1)}, \\
\mathbf{p}^{\prime} & =\mathbf{p}_{i}^{(1)}-\mathbf{p}_{f}^{(2)}=\mathbf{p}_{i}^{(1)}-\mathbf{p}_{i}^{(2)}-\mathbf{p} .
\end{aligned}
$$

For energy and momentum we have the conservation laws

$$
\begin{gathered}
\mathbf{p}_{i}^{(1)}+\mathbf{p}_{i}^{(2)}=\mathbf{p}_{i}^{(1)}+\mathbf{p}_{i}^{(2)}, \\
c \sqrt{ }\left(M^{2} c^{2}+p_{i}^{(1 / 2)}+c \sqrt{ }\left(M^{2} c^{2}+p_{i}^{(2) 2}\right)\right. \\
=c \sqrt{ }\left(M^{2} c^{2}+p_{i}^{(1 / 2)}+c \sqrt{ }\left(M^{2} c^{2}+p_{i}^{(2) 2}\right)\right.
\end{gathered}
$$

or

$$
E_{i}^{(1)}+E_{i}^{(2)}=E_{j}^{(1)}+E_{j}^{(2)} .
$$

The second term in (2) is caused by the Pauli principle. After the collision it is impossible to decide which of the particles is the impinging one. So we have two terms, the first indicating that the particle with lower energy is particle (2), the second that it is particle (1). In the first case the momentum exchange is small, in the second large. In the latter case the impinging neutron communicates most of its energy to the knocked-on particle. So we may use our formula only up to half the largest possible energy transfer. Then the energies of both particles are equal after the collision, the corresponding momentum being $\mathbf{p}_{\mathbf{1}}$. From (4) and (5) follows

$$
E_{i}^{(1)}+E_{i}^{(2)}=2 c \sqrt{ }\left(M^{2} c^{2}+p_{i}^{2}\right) .
$$


The second term in (2) is the term for large energy exchange. We shall call it the exchange term.

These differences in the momentum exchange will also appear in the expression for $d n_{j}$. We introduce polar coordinates in momentum space with $\mathbf{p}_{i}^{(1)}$ as polar axis and counting the azimuths from the plane $\left(\mathbf{p}_{i}^{(1)}, \mathbf{p}_{i}^{(2)}\right)$. The coordinates of $\mathbf{p}$ are $p, \theta$ and $\varphi$ with $\zeta=\cos \theta$. For the following we put $\mathbf{p}_{i}^{(2)}=0$. Then

$$
p_{f}^{(1) 2}=p_{i}^{(1) 2}+p^{2}-2 p p_{i}^{(1)} \zeta .
$$

For a given momentum exchange $p, d n_{f}$ is $\left.{ }^{*}\right)$

$$
d n_{f}=\frac{V}{(2 \pi \hbar)^{3}} p d p d \varphi \cdot \frac{c}{v_{i}^{(1)}}\left(1-\frac{\sqrt{ }\left(M^{2} c^{2}+p^{2}\right)-M c}{\sqrt{ }\left(M^{2} c^{2}+p_{i}^{(1) 2}\right)}\right) .
$$

Of course, this formula is also valid for large momentum exchange. The exchange term, however, is a function of $\mathbf{p}^{\prime}=\mathbf{p}_{i}^{(1)}-\mathbf{p}$, and for the integrations it is desirable to have $d n_{f}$ as a function of $\mathbf{p}^{\prime}$. Denoting the coordinates of $\mathbf{p}^{\prime}$ by $p^{\prime}, \theta^{\prime}$ and $\varphi^{\prime}$ with $\zeta^{\prime}=\cos \theta^{\prime}$, we find

$$
d n_{f}=-\frac{V}{(2 \pi \hbar)^{3}} p^{\prime} d p^{\prime} d \phi^{\prime} \frac{c}{v_{i}^{(1)}}\left(1-\frac{\sqrt{ }\left(M^{2} c^{2}+p^{\prime 2}\right)-M c}{\sqrt{ }\left(M^{2} c^{2}+p_{i}^{(1) 2}\right)}\right) .
$$

The form of this equation is the same as that in $\mathbf{p}$. Then the cross section is:

$$
\begin{aligned}
d \bar{s}=B \frac{c^{2}}{v_{i}^{(1) 2}}( & \left.\ldots \frac{2}{1}+\frac{2}{+(p / \hbar x)^{2}}\right)^{2}\left(1-\frac{\sqrt{ }\left(M^{2} c^{2}+p^{2}\right)-M c}{\sqrt{ }\left(M^{2} c^{2}+p_{i}^{(1) 2}\right)}\right) \frac{p}{\hbar^{2} \varkappa^{2}} d p d \varphi \\
& +\left(\text { similar term in } p^{\prime} \text { and } \varphi^{\prime}\right),
\end{aligned}
$$

where

$$
B=0.52 \frac{J^{2}}{c^{2} \hbar^{2} \varkappa^{4}}
$$

The first term must be integrated over $p$ from 0 up to $p_{1}$, the second over $p^{\prime}$ from $p_{i}$ to $p_{i}^{(1)}$ (cf. equ. (2)).

Introducing

$\beta=M c / \hbar \varkappa, \quad \alpha=p_{i}^{(1)} / \hbar \varkappa, u=p / \hbar \varkappa, u^{\prime}=p^{\prime} / \hbar \varkappa, u_{1}=p_{i} / \hbar \varkappa, \gamma=\sqrt{ }\left(\alpha^{2}+\beta^{2}\right)$ we have:

$$
\begin{aligned}
d \bar{s}=B & \frac{\gamma^{2}}{\gamma^{2}-\beta^{2}} \frac{4}{\left(1+u^{2}\right)^{2}} \frac{\gamma+\beta-\sqrt{ }\left(u^{2}+\beta^{2}\right)}{\gamma} u d u d \varphi \quad \text { with } u<u_{1} \\
& +\left(\text { similar term in } u^{\prime} \text { and } \varphi^{\prime}\right) \text { with } u^{\prime}>u_{\xi} .
\end{aligned}
$$

*) Cf. Rose nfeld loc. cit. 
We can combine these two terms, if there is no function of $p$ to be integrated. So we have for the total cross section

$$
\bar{s}=2 \pi \int_{u=0}^{u=a} \frac{d \bar{s}}{d u} d u .
$$

For some calculations it is desirable to take the energy, expressed in units $\delta=\sqrt{ }\left(\beta^{2}-1\right)$, as the independent variable. Putting

we find

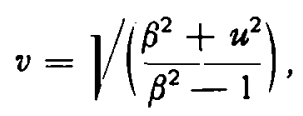

$$
d \bar{s}=B \frac{\gamma^{2}}{\gamma^{2}-\beta^{2}} \frac{1}{\delta^{2}} \frac{4}{\left(v^{2}-1\right)^{2}} \frac{\gamma+\beta-\delta v}{\gamma} \cdot v d v d \varphi .
$$

The limits are $\beta / \delta,(\beta+\gamma) / 2 \delta=v_{1}$ and $(\beta+\gamma) / 2 \delta=v_{1}, \gamma / \delta$.

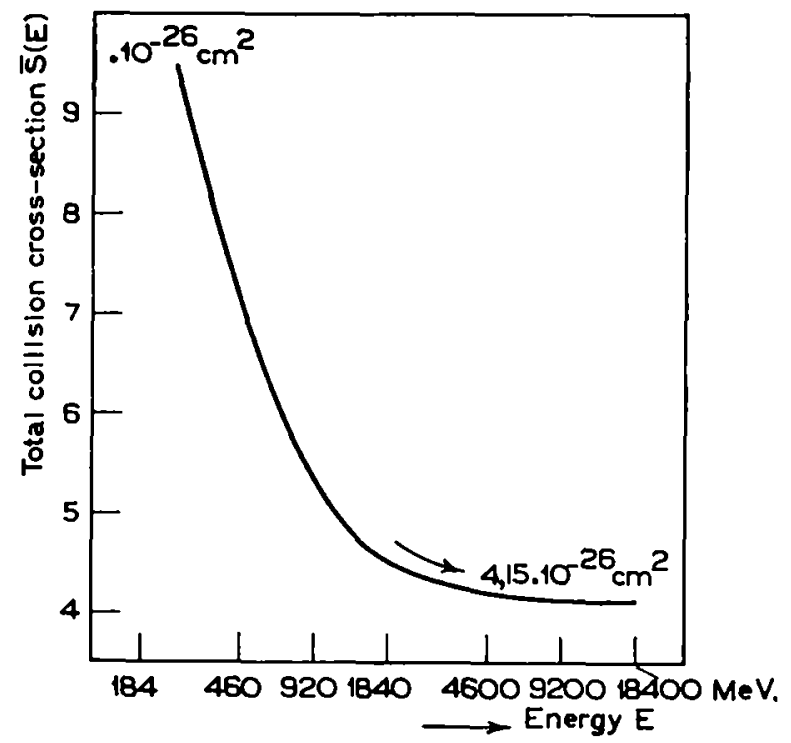

Fig. 1. The mean total collision cross-section for a given kinetic energy of one of the particles, the other being at rest.

Figure 1 shows $\bar{s}$ as a function of the kinetic energy of the impinging neutron. The limiting value of $\bar{s}$ for large energy is

$$
4.15 \times 10^{-26} \mathrm{~cm}^{2} \text { if } x^{-1}=2 \times 10^{-13} \mathrm{~cm} \text {. }
$$


We have also computed the relative probability of a collision with a given energy transfer, i.e. the ratio of $\int_{E_{2}}^{E_{1}} d \bar{s}$ to $\bar{s}$. In the table we give the probability in per cents of the total cross section for a collision with an energy transfer between some important limits.

TABLE I

\begin{tabular}{|c|c|}
\hline \multicolumn{2}{|c|}{$\begin{array}{l}\text { The probability of an energy } \\
\text { transfer in a given interval for a } \\
\text { collision of two nucleons in par } \\
\text { cents of the total cross-section }\end{array}$} \\
\hline Interval & $\begin{array}{l}\text { Probability } \\
\text { in } \%\end{array}$ \\
\hline $46-92 \mathrm{MeV}$ & 5.7 \\
\hline $92-138$, & 1.9 \\
\hline $138-184$, & 0.95 \\
\hline$>184$ & 2.59 \\
\hline
\end{tabular}

These probabilities are practically independent of the energy of the impinging nucleon, provided that this energy is higher than $400 \mathrm{MeV}$.

III. The energy distribution of the knocked-out particles. Instead of directly comparing the computed energy distribution with the experimental data, it is better to compare the ratios of the number of nucleons with an energy larger than a given energy $E$ ( $E$ is the non relativistic kinetic energy of the knocked nucleon after the collision). This number is proportional to $\bar{s}(E)=\int_{E}^{\infty} d \bar{s}$. Using the general formulae for $\bar{s}(E)$ given by $\mathrm{R}$ os e $\mathrm{n}$ e ld, we find for the meson potential

$$
\bar{s}(E) \sim \int_{H(\eta+1)}^{H(\eta-1)}\left[2 H^{2}\left(\eta^{2}+1\right)-x^{2}-\frac{H^{4}\left(\eta^{2}-1\right)^{2}}{x^{2}}\right] \frac{2}{1+x^{2}} d x
$$

with $H=p_{m}^{0^{0}} / \hbar \varkappa$ and $\eta=\sqrt{ }\left(E / E_{m}^{0}\right), E_{m}^{0}$ being the nonrelativistic maximum kinetic energy of the zero point distribution and $p_{m}^{0}$ the corresponding momentum. Performing the integration we find with the substitution $2 H t=1+H^{2}\left(\eta^{2}-1\right)$ :

$$
\bar{s}(E) \sim\left(t^{2}+1\right) \operatorname{arc} \operatorname{cotg} t-t .
$$


This formula is valid only if the contribution of collisions with high energy transfer is negligible, for only then we may take $\infty$ instead of $\alpha$ as upper limit of integration. The energy $E$ inside the nucleus corresponds with an energy $E_{0}=E-\left(E_{m}^{0}+\varepsilon\right)$ outside, $\varepsilon$ being the average binding energy per nucleon. The values used are $E_{m}^{0}=24.2 \mathrm{MeV}$ and $\varepsilon=8.6 \mathrm{MeV}$.

It is, however, impossible to measure the number of nucleons $n\left(E_{0}\right)$ with an energy outside the nucleus larger than $E_{0}$ with accuracy. Very high energies will not be recorded in the photographic emulsion, while nucleons of $100-200 \mathrm{MeV}$ easily escape attention. So we can only compare the number of particles in certain intervals. On the other hand this is also desirable from a theoretical point of view, in order to eliminate the contribution of the upper limit of integration, for which we took $\infty$. If $E_{l}$ is an energy, which is certainly fully recorded in the emulsion, we have to compare $\bar{s}\left(E_{\mathrm{c}}\right)-$ $-\bar{s}\left(E_{l}\right)$ with $n\left(E_{0}\right)-n\left(E_{l}\right)$. Then the choice of the upper limit of $p$ is of no importance, except for the contribution of the exchange term. If the energy of the impinging particle is very high, we may neglect this contribution which is $\bar{s}\left(E_{i}^{(1)}-E_{l}\right)-\bar{s}\left(E_{i}^{(1)}-E_{c}\right)$.

The table for $\bar{s}(E)$ is computed for three values of $\varkappa^{-1}$, namely $1.8 \times 10^{-13} \mathrm{~cm}, 2.0 \times 10^{-13} \mathrm{~cm}$ and $2.2 \times 10^{-13} \mathrm{~cm}$, neglecting the exchange contribution. A factor $1.56 \pi J^{2} / H v_{i}^{(1) 2} \hbar^{2} x^{4}$ has been omitted in $\bar{s}(E)$. From it we calculated the relative numbers of nucleons $\sigma=\left\{\bar{s}\left(E_{0}\right)-\bar{s}\left(E_{l}\right)\right\} /\left\{\bar{s}(10 \mathrm{MeV})-\bar{s}\left(E_{l}\right)\right\}$ with an energy between $E_{c}$ and $E_{l}$ for two values of $E_{l}$ resp. $110 \mathrm{MeV}$ and $60 \mathrm{MeV}$. We have also computed these relative numbers for a Gauss potential, assuming $*^{-1}=2.0 \times 10^{-13} \mathrm{~cm}$. The experimental ratios are computed from measurements by Perkins (1947) and W a m b c her (1940) and refer respectively to 46 and 194 tracks of $E_{0}>10 \mathrm{MeV}$.

From table II it is clear that the three values chosen for $x^{-1}$ satisfactorily agree with the figures of $\mathrm{Wambacher}$. It is, however, impossible to make a choice for $x^{-1}$, different factors being responsible for this. First stars with a small number of proton tracks were excluded from the statistics. (This is necessary because stars with a small number of tracks might originate from light nuclei.) A large number of single tracks and of stars, attributed to light nuclei, must originate from a collision with a heavy nucleus. The energy of the impinging neutron was supposed to be larger than $200 \mathrm{MeV}$. Only in that case may the exchange contribution be 
neglected. The latter will give only a slight increase in the computed number of particles, especially for the higher energies if the energy of the impinging neutron is about $2 E_{l}$. Further the effect of secondary collisions has been neglected, which collisions would result in a increase in the number of low energy particles at the cost of those of high energy. Finally it is important to remark that a different choice of $E_{m}^{0}$ gives about the same percentage change in the best value of $x^{-1}$. These small differences make it impossible to make a choice of $x^{-1}$, even with the help of a large number of observations.

TABI,E II

\begin{tabular}{|c|c|c|c|c|c|c|c|c|}
\hline \multirow{3}{*}{$\begin{array}{c}E_{\mathrm{o}} \\
(\mathrm{MeV})\end{array}$} & \multicolumn{3}{|c|}{$\bar{s}\left(E_{0}\right)$} & \multicolumn{5}{|c|}{$\sigma\left(E_{l}=110 \mathrm{MeV}\right)$} \\
\hline & \multicolumn{3}{|c|}{$x^{-1}=$} & \multicolumn{3}{|c|}{$x^{-1}=:$} & \multirow{2}{*}{ '" } & \multirow{2}{*}{ Ciauss } \\
\hline & 1.8 & 2.0 & 2.2 & 1.8 & 2.0 & 2.2 & & \\
\hline 10 & 0.571 & 0.548 & 0.523 & 100 & 100 & 100 & 100 & 100 \\
\hline 20 & 0.4365 & 0.4115 & 0.387 & 69.3 & 68.1 & 67.1 & 48 & 56.0 \\
\hline 30 & 0.350 & 0.3275 & 0.303 & 49.6 & 48.5 & 46.5 & 24 & 32.7 \\
\hline 40 & 0.292 & 0.270 & 0.2455 & 36.4 & 35.0 & 32.7 & 19.5 & 17.3 \\
\hline 50 & 0.2495 & 0.229 & 0.211 & 26.8 & 25.5 & 24.3 & 15 & 10.3 \\
\hline 60 & 0.216 & 0.198 & 0.184 & 19.1 & 18.2 & 17.8 & 8.6 & 5.3 \\
\hline 70 & 0.192 & 0.177 & 0.163 & 13.6 & 13.3 & 12.7 & 6.5 & 2.1 \\
\hline 90 & 0.157 & 0.144 & 0.132 & 5.7 & 5.6 & 5.2 & 4.3 & 0.9 \\
\hline 110 & 0.132 & 0.120 & 0.1105 & & & & & i: \\
\hline \multirow{3}{*}{$\begin{array}{c}E_{0} \\
\left.(. \mathrm{Me}\rangle^{\prime}\right)\end{array}$} & \multicolumn{8}{|c|}{$\sigma\left(E_{1}=60 \mathrm{Mel}\right)$} \\
\hline & \multicolumn{3}{|c|}{$x^{-1}=$} & & \multirow{2}{*}{ I } & \multirow{2}{*}{11} & $\|$ & \multirow{2}{*}{ Gallss } \\
\hline & 1.8 & 2.0 & 2.2 & ? & & & $i$ & \\
\hline 10 & 100 & 100 & 100 & & 100 & 1 & & 100 \\
\hline 20 & 62.2 & 61.2 & 59 & & 43 & & & 53.5 \\
\hline 30 & 37.8 & 37.1 & 35 & $i$ & 16.7 & & & 28.4 \\
\hline 40 & 21.4 & 20.8 & 18 & 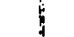 & 11.9 & & & 17.7 \\
\hline 50 & 10.0 & 8.9 & & ) & 7.2 & & 8 & 5.2 \\
\hline
\end{tabular}

About the directions of the knocked-out nucleons we can say the following:

If $E_{i}^{(1)}$ is very large, the direction of the knocked nucleon will lie in a plane perpendicular to the direction of the neutron. If there are secondary collisions the tracks in the emulsion may considerably deviate from this plane. So we expect the larger energy tracks to lie in the perpendicular plane, the slower nucleons having a more isotropic distribution. If the neutron energy is about $200 \mathrm{MeV}$ or 
less, the only thing we may expect is, on the average, a slight preference for the direction of the incoming neutron.

IV. Energy loss in nuclear matter. On its way through a nucleus a fast nucleon loses kinetic energy owing to collisions. According to a theorem by Willi a m s the mean energy loss per unit path is the same as though the nucleons inside the nucleus were at rest. So we have to integrate $p$ from 0 to $p_{\xi}$ and $p^{\prime}$ from $p_{t}$ to $p_{i}^{(1)}$.

The functional form of the energy loss in $p$ is different from that in $p^{\prime}$, viz.

$$
c \sqrt{ }\left(M^{2} c^{2}+p^{2}\right)-M c^{2} \text { and } c \sqrt{ }\left(M^{2} c^{2}+p_{i}^{(1) 2}\right)-c \sqrt{ }\left(M^{2} c^{2}+p^{2}\right) \text {. }
$$

After performing a transformation to the variables $v, \alpha, \beta, \gamma$ and $\delta$ they read $c \hbar x(\delta v-\beta)$ and $c \hbar x(\gamma-\delta v)$, writing $v$ for $v^{\prime}$.

The energy loss per unit length is $d E_{i}^{(1)} / d s=-(A / V) \int E d \bar{s}$, $E$ being the energy loss and $(A / V)$ the density of nuclear matter.

$$
\begin{gathered}
\frac{d E_{i}^{(1)}}{d s}=-\frac{A}{V} c \hbar x B \frac{\gamma}{a^{2} \delta^{2}}\left[\int_{0}^{2 \pi} \int_{\beta^{\prime} \delta}^{(\beta+\gamma) / 2 \delta} \frac{4}{\left(v^{2}-1\right)^{2}}(\gamma+\beta-\delta v)(\delta v-\beta) v d v d \varphi\right. \\
\left.+\int_{0}^{2 \pi} \int_{(\beta+\gamma) / 2 \delta}^{\gamma \delta} \frac{4}{\left(v^{2}-1\right)^{2}}(\gamma+\beta-\delta v)(\gamma-\delta v) v d v d \varphi\right] \\
=-(A / V) c \hbar x B\left(2 \pi \gamma / a^{2} \delta\right)\left[(3 \gamma+3 \beta-4 \delta) \log \left\{\frac{1}{4}(\gamma+\beta)^{2}-\delta^{2}\right\}\right. \\
-6(\gamma+\beta) \log \left\{\frac{1}{2}(\gamma+\beta)+\delta\right\}-(2 \gamma+\beta-2 \delta) \log \left(\gamma^{2}-\delta^{2}\right) \\
+(4 \gamma+2 \beta) \log (\gamma+\delta)+(2 \gamma+4 \beta) \log (\beta+\delta)] .
\end{gathered}
$$

For $x^{-1}=2 \times 10^{-1} \mathrm{~cm}$ we find $\beta=9.40, \delta=9.35$ and $\beta / \delta=1.0057$.

Putting $P=\gamma / \delta$ we have $d E_{i}^{(1)} / d s=-(A / V) C /(P)$

where $C=(2 \pi / \delta) c \hbar \varkappa B=1.04 \pi J^{2} / \delta c \hbar x^{3}$

and

$$
\begin{array}{r}
f(P)=P\left(P^{2}-1.0114\right)^{-1}\left[(3 P-0.9829) \log \left(\frac{1}{2} P-0.4971\right)\right. \\
-(3 P+7.017) \log \left(\frac{1}{2} P+1.503\right)+(2 P+3.006) \log (P+1) \\
-(2 P-0.994) \log (P-1)+(2 P+4.023) \log 2.006 \\
+(2 P+0.023) \log 9.35] .
\end{array}
$$


Graph 2 shows this function $f(P)$ (using the $\log _{10}$ ) and the energy loss per collision, as a function of the kinetic energy of the impinging neutron. The transformation factor to $\log _{e}$ and to $\mathrm{MeV}$ may be taken together with $C$. Then $C=50.4 \times 10^{-26} \mathrm{MeV}$.

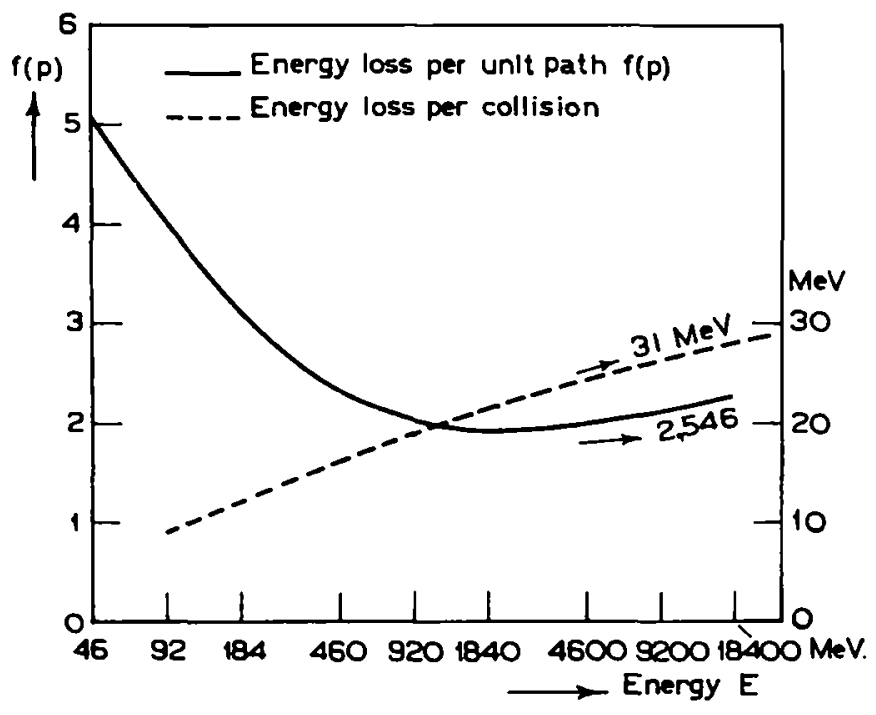

Fig. 2. The energy loss per unit path in nuclear matter $f(P)$ in units $(A / V) \times 2.39 \pi J^{2} / \delta c \hbar x^{3}$ and the mean energy loss per collision of a nucleon with kinetic energy $E$.

From the graph one sees that the energy loss of a nucleon with kinetic energy larger than $400 \mathrm{MeV}$ is practically constant. Hence it is permitted to write for the total energy loss of fast nucleon running through a nucleus as

$$
\Delta E=\Delta s .(A / V) f(P) \times 50 \times 10^{-26} \mathrm{MeV}
$$

For $\mathrm{Ag}$ and $\mathrm{Br}(A / V) \Delta s \cong 10^{26}$. So the mean energy loss of a very fast nucleon has the limit $130 \mathrm{MeV}$. This value would seem too low to explain the frequent occurrence of stars with an energy beyond $300 \mathrm{MeV}$.

To get an idea of the probability of large star energies in the above theory we have taken the following device. The mean number of collisions within a nucleus of $\mathrm{Ag}$ or $\mathrm{Br}$ is $\bar{s}(A / V) \Delta s(\bar{s}=$ total cross section). From figure 1 it follows that $\bar{s} \cong 4.5 \times 10^{-26} \mathrm{~cm}^{2}$ for large energies. Further $(A / V) \Delta s \cong 10^{26} \mathrm{~cm}^{-2}$. So there are 
about 4.5 primary collisions. For high energy particles the number is about 4, which number we shall use. With the help of table I we compute the probabilities for two and three collisions with an energy exchange in the intervals (1) 46-92 MeV, (2) $92-138 \mathrm{MeV}$ and (3) 138-184 MeV. Only these intervals are important, for nucleons with smaller energy do not contribute much while high energy particles will escape without being recorded, the probability for secondary collisions with large energy transfer being of the order of $5 \%$. So we get a table for the probability of two collisions in interval (1). (2) or (3); one collision in (1) and one in (2) and so on. Taking for each interval a mean value of the energy transfer, we get a list of probabilities for collisions with a certain mean energy transfer. Adding all probabilities with energy transfer within certain intervals we find roughly the following probabilities for an energy transfer to the nucleus:

$$
\begin{array}{lll}
150-250 \mathrm{MeV} & 2 \% \\
250-350 \quad, & 0.1 \% \\
350-450 \quad, & 0.01 \%
\end{array}
$$

Having no figures at my disposal, I could not make a comparison with experimental data. As the probability for collisions with a small energy transfer is great and these collisions will not be recorded as stars in the emulsion, the absolute value of the above figures is not very important. Only the relative values may be compared with statistics of star energies.

If the energy of the impinging nucleon is of order of $300-400 \mathrm{MeV}$, these figures will be higher because after two collisions of about 100 $\mathrm{MeV}$ the remaining energy of the neutron is also of this order.

Another way to explain the occurrence of high energy stars is the hypothesis that the impinging particle is a meson or an $\alpha$-particle. In the first case the energy will mainly originate from the mass energy of the meson. The loss of mass energy in two steps seems favourable, this energy being distributed among several nucleons. In the second case we can roughly say that the collision cross section of an $\alpha$-particle is a few times that of a nucleon with $\frac{1}{4}$ of the kinetic energy of the $a$-particle. The mean energy loss is then a few times that of a nucleon, i.e. the total energy, if the energy of the a-particle is about $500 \mathrm{MeV}$. It is, however, impossible that this particle forms a part of the cosmic radiation. We must therefore 
assume that it originates in a collision of a constituent of the cosmic radiation with the same or a neighbouring nucleus. These possibilities are still to be examined.

I wish to express my thanks to prof. L. R o s e $\mathrm{n}$ f e l d for many useful discussions.

Received June 24th 1948.

E. B a g g e, Ann. Physik 35, 118, 1939.

W. H e ise n ber $\mathbf{g}$, Leipz. Ber. 88, 369, 1937.

G. Ort ner, Wiener Ber. 140, 259, 1940.

D. Perkin s, Nature 160, 229, 1947.

L. R os e n f e ld, Nuclear Forces, Amsterdam 1948.

H. W a m b a c her, Wiener Ber. 1AN, 157, 1940. 